\title{
The transient interaction of plates with extreme water waves: effects of deformability and hull cavitation
}

\author{
Sh. U. Galiev \& R. G. J. Flay \\ Department of Mechanical Engineering, The University of Auckland, \\ Private Bag 92019, Auckland 1142, New Zealand
}

\begin{abstract}
The cavitation aspect of the transient interaction of deformable surfaces with water waves is considered. This problem is examined using an experimental approach by creating a pressure wave in water and impacting it on metal plates. Such loadings can occur from underwater explosions or from large surface waves, and are important for the design of merchant and naval ships, and offshore structures. Often these loadings cannot be predicted very accurately. In particular, the uncertainties in the loadings are associated with cavitation that is generated during the transient water-deformable surface interaction process.

Keywords: extreme water waves, hull cavitation, deformability, structure-fluid interaction.
\end{abstract}

\section{Introduction}

It is common for mid-ocean storm waves to reach $7 \mathrm{~m}$ in height, and in extreme conditions such waves can reach heights of $15 \mathrm{~m}$. However, during recent years researchers observed the existence of vastly more massive waves - veritable monsters up to $30 \mathrm{~m}$ in height. Such waves were said to consist of an almost vertical wall of water. It has been confirmed that these monsters are much more common than mathematical probability theory would predict using a Rayleigh distribution of wave heights. In fact, they seem to occur in all of the world's oceans many times every year [1-4].

The existence of these extreme waves makes it important to re-examinee some fundamental ideas of merchant ship design that were earlier developed 
[5-7]. In particular, the experience from designing underwater naval ships (submarines), which are required to retain a high standard of operational effectiveness under explosion attack, may be used.

Underwater weapons usually explode close to the submarine hull (Fig. 1). With the arrival of the shockwave generated by the explosion, the pressure rises very quickly to a peak value and then the peak is followed by a decay. The time for the pressure to decay to $e^{-1}$ of its maximum value is on the order of a millisecond $(0.001 \mathrm{~s})$, after which the pressure decay rate becomes slower than the exponential rate. The results of the underwater explosions depend on the amplitude and duration of the loading by the shock wave, and also on the character of the shock wave - structure interaction. In particular, due to deformability of the structure the shockwave can be reflected as a rarefaction wave. Cavitation can occur in the water if the pressure in the rarefaction wave drops below some critical value.

This process is understood for underwater explosions [7-9]. The present paper examines whether similar processes may accompany the interaction of extreme surface waves with the hulls of ships.

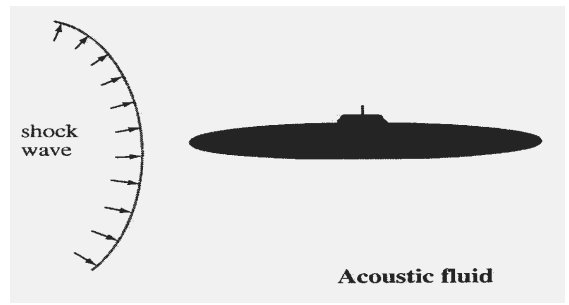

Figure 1: Sketch of shock wave induced by an underwater explosion impinging on a submarine (from [9]).

The first goal of this paper is to show that the action of extreme surface waves can generate shockwave-like pressures on a ship's hull. The second goal is to study the cavitation interaction of metal plates with shockwaves in water.

\section{Some important features of extreme wave/rigid surface interaction}

In this section the ship's surface is assumed to be an absolutely rigid wall loaded by ocean waves. Results from [10] are used to illustrate the pressure developed on such walls.

The force exerted by sea waves on a vertical wall depends, for any given shape of approaching wave, on the thickness of water normal to the wall (see Fig. 2). The force is quite small if the water layer is very thick, and is again quite small if the ambient water level is well below the foot of the wall. But there is a certain water thickness for which the force on the wall is a maximum (Fig. 2d). Higher shock pressures may result, but they last for only $0.01 \mathrm{~s}$. The 


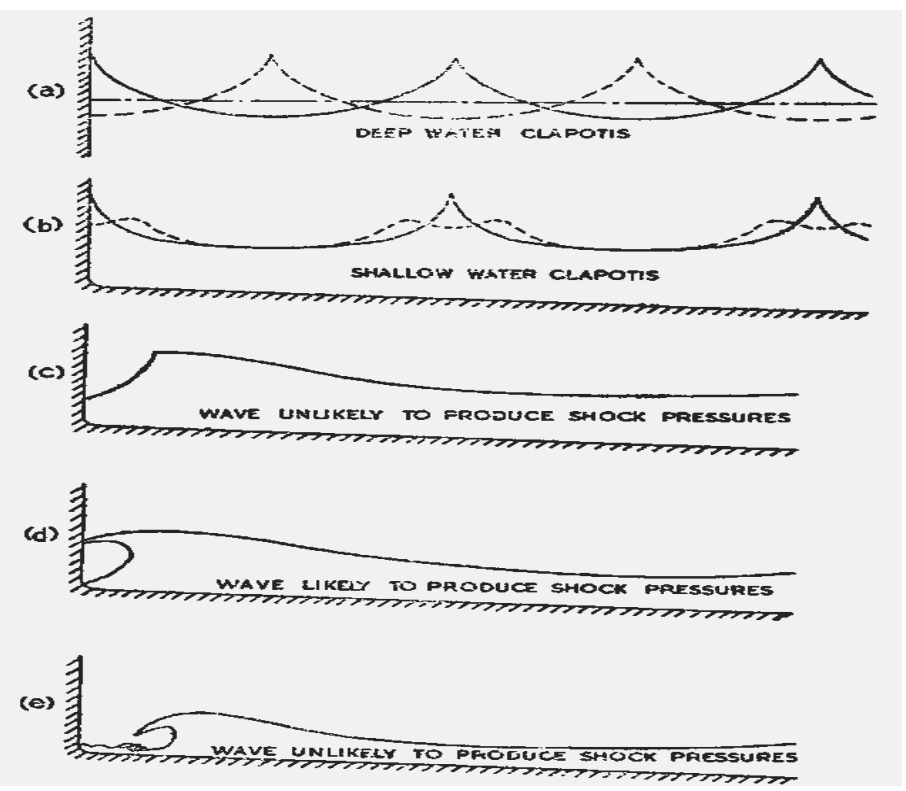

Figure 2: $\quad$ Types of wave action to which a wall may be subjected, depending on the thickness of the water layer in front of the wall. The situation depicted in (d) gives rise to the highest pressure (from [10]).

pressures generated depend greatly on the irregularity of waves [10]. In particular the shock pressures are affected by this irregularity, making the prediction of their magnitude almost impossible.

In these experiments [10] the pressure was measured by piezo-electric gauges at points located $0.64 \mathrm{~m}$ and $1 \mathrm{~m}$ below mean sea level, and at a point located $0.33 \mathrm{~m}$ above mean sea level. A few waves, never more than five in a hundred, generated high pressures. The pressures reached their maxima in a time as short as $0.005 \mathrm{~s}$ or as long as $0.05 \mathrm{~s}$. The maximum pressure at the lowest level was $0.68 \mathrm{MPa}(14,300$ pounds per square foot) (see Fig. 3), at the intermediate level was $0.29 \mathrm{MPa}$, while at the highest-level pressure shocks were very seldom recorded.

The profile of the pressure curves can be described by the exponential function. The duration of the loading may be of the order of $0.005 \mathrm{~s}$. Thus the profile and the duration of the pressure time histories from violent surface waves are exactly the same as from underwater shock waves.

It was observed that sometimes a pocket of air was trapped between the wall and the concave front face of the advancing wave, and it was verified that shock pressures only occurred when the advancing wave enclosed a pocket of air between itself and the wall. When the pocket of air was "thin" the pressure was greatest. When the pocket of air was thicker so that its dimension normal to the wall was similar to its height the pressure rise due to the wave was negligible. 
Even these high pressures are only one-sixth of what is theoretically possible and the duration of the impact, short though it is, is ten times greater than can be calculated from water-hammer theory, assuming that the pocket is infinitely thin [10]. The reduction of the pressures generated on sea walls and the increase in duration from what is theoretically possible, is in accordance with the idea of a trapped air pocket cushioning the shock (pp 142-145, [10]). We emphasise that the data presented above from [10] agree well with those resulting from more recent research [11,12]. In particular, Peregrine [11] noted that 'In the laboratory violent peak pressures act for times of approximately 1 millisecond, and peak durations range from 10 to 100 milliseconds in the field'.

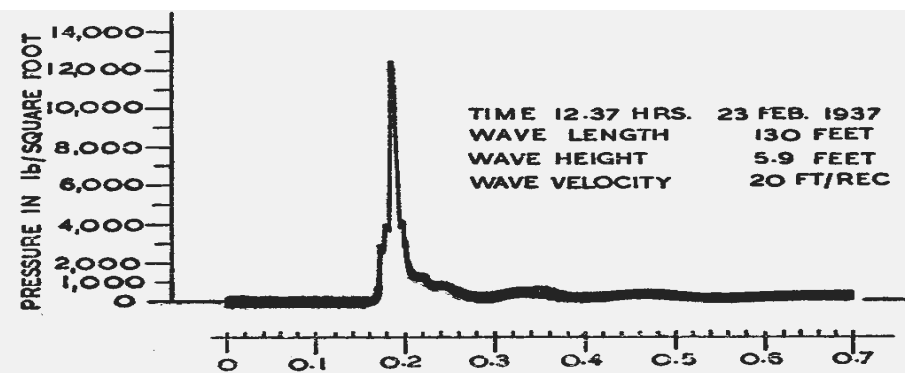

Figure 3: $\quad$ Pressure on a vertical wall caused by waves breaking (from [10], time in seconds).

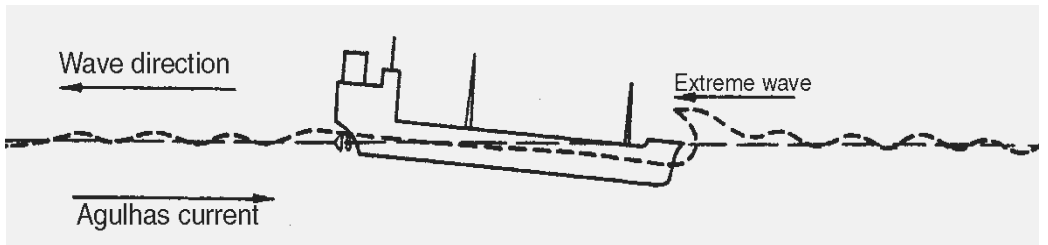

Figure 4: Extreme wave collision with the Taganrogsky Zaliv described by a witness (from [13]).

The results discussed above show that breaking waves and waves having very step fronts can generate violent pressures on solid surfaces. The steepness of the wave determines the pressure. Apparently this conclusion is also valid for ocean waves acting on the ships, according to observations from sailors (Fig. 4) [13].

In a "collision" between a wave and a ship the outcome depends mainly on the dimensions and mass of the ship, the height of the wave and the steepness of its front. The local damage to the ship's surface depends, of course, on the strength of ship elements and the interaction of these elements with the impact of the water. The character of the interaction changes from one element to another. The effect of the trapped atmospheric air may be very important. This effect perhaps explains the rarity of catastrophic failure of ships that are hit by extreme ocean waves. Indeed, due to the trapped atmospheric air the ship is really loaded by an air-water mixture instead of practically incompressible water (water- 
hammer). In particular, the gas-water mixture may be generated from hull cavitation. This cavitation may be considered as an analogue of the trapped atmospheric air. However, in contrast with the air/water mixture, cavitation limits the reduction of the water pressure. As a result, cavitation can increase the common pressure on the deforming surface and may determine the result of the ship hull-extreme surface wave interaction. The authors emphasise that because of the trapped atmospheric air on cavitation, there are large uncertainties in the determination of real loads from water waves on structural elements of ships. Therefore any experimental data that enlighten this issue concerning the interaction of extreme waves with deformable structural elements are clearly useful in helping this problem to be understood. The next section considers results from an experimental investigation of cavitation interaction between metal plates and underwater pressure waves.

\section{Plate-underwater wave interaction}

Tests were conducted using an apparatus whose principal features are presented in Fig. 5(a). The fundamental principle used in the apparatus is the conversion of the kinetic energy of a rapidly moving mass (a flat piston) into water pressure energy. The piston was accelerated by a compressed gas in a cylinder (radius $R$ $=55 \mathrm{~mm}$ ).
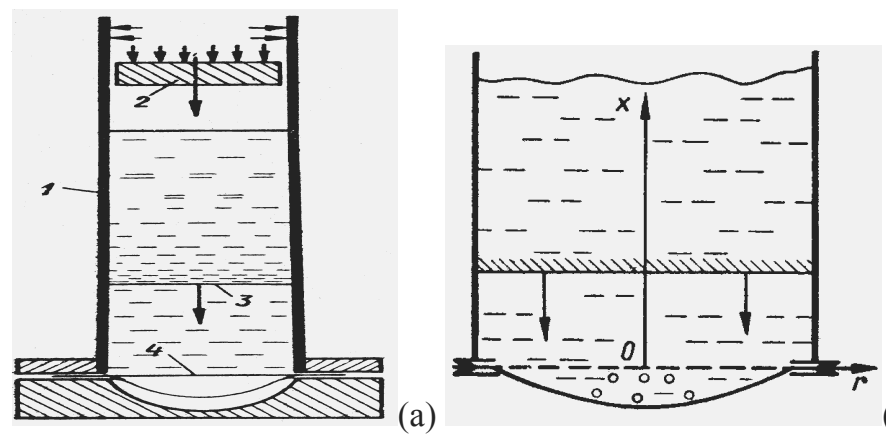

Figure 5: $\quad$ Sketches of the experimental apparatus (a) and plate-shock wave interaction (b).

The experimental apparatus consists of a vertical cylindrical metal tube (1) having an inner diameter of $112 \mathrm{~mm}$. The tube wall thickness is $20 \mathrm{~mm}$. The acoustical sound speed calculated for water in the tube was $1265 \mathrm{~m} / \mathrm{s}$. The plane piston (2) impacts the water surface and causes a compression wave (3). The tube bottom is closed by a circular plate that is clamped around its edge. This plate can be replaced during the experiments in order to examine its properties (thickness $h$ and stiffness) on the resulting pressures that are developed. The compression wave deforms the plate as shown in the sketch. The plate is shown in its undeformed and deformed states in Fig. 5(a)(4) and 5(b) respectively. The height of the water column was $500 \mathrm{~mm}$. An air layer ahead of the piston was 
compressed during its acceleration. This diminished the wave amplitude in the water by about half compared to what it would have been in the absence of an air layer.

The experiments studied the case of small (elastic) deformations of the plate when the mass of the piston was $13 \mathrm{~kg}$ and the impact velocity of the piston onto the water surface was kept constant at $1.8-2.0 \mathrm{~m} / \mathrm{s}$. The pressures were measured at different points in the water using piezoelectric pressure sensors and were recorded on an oscilloscope screen. The piezoelectric pressure sensors and displays had previously been calibrated statically and dynamically and both calibrations gave identical results.

An example oscillogram of the pressure measured on the surface of a steel plate with thickness $h=20 \mathrm{~mm}$ is shown in Fig. 6 . It can be seen that the pressure increases rapidly and then decays in an exponential-type manner. This pressure trace, rising to $1.25 \mathrm{MPa}$ and with a wavelength of $2.5-3 \mathrm{~m}$, is the incident wave and is the same for all tests with elastic plates discussed below.

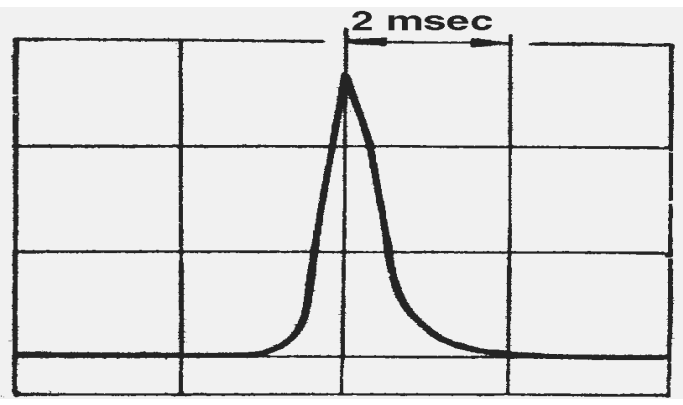

Figure 6: An oscillogram of the pressure in the incident wave.

Results from aluminium alloy plates with thicknesses $h=8$, 4, and $2 \mathrm{~mm}$ are shown in Figs. 7(a), (b) and (c) respectively. The pressure oscillograms measured on the tube wall at points $255 \mathrm{~mm}$ and $55 \mathrm{~mm}$ from the plate surface correspond to curves I, and II respectively. The left hand graphs have a 2 milli-s time scale, and the right hand graphs have a 0.5 milli-s timescale. It can be seen that a reduction in the plate thickness results in a radical change in the pressure curves with an increase in the interaction time.

In analysing the oscillograms presented in Fig. 7, we will consider primarily the curves obtained by sensor II close to the plate. These curves consist of the first peak, a pressure drop section, and a second peak, which is somewhat longer. The first peak results from the pressure occurring at the very beginning of the interaction when the plate just starts to move. The interaction is evident here in that the peak amplitude depends on the plate thickness and is less than the maximum pressure of 1.25 MPa in the incident wave (Fig. 6).

The pressure remains positive in the pressure drop section for the $8 \mathrm{~mm}$ thick plate (a). Cavitation occurs in the water for the 4 and $2 \mathrm{~mm}$ thick plates (b) and (c) respectively. The pressure then rises, which is explained by the collision between the water volume and the plate when the cavitation zone near the plate 
collapses. The amplitude of the second peak is close $(h=8 \mathrm{~mm}$ (a)) to, or exceeds ( $h=4 \mathrm{~mm}$ (b) and $h=2 \mathrm{~mm}$ (c)) the magnitude of the first peak, and depends less on $h$. The length of the pressure decay section and of the second peak is related to deceleration of the plate by elastic forces and the velocity of its reverse motion, i.e., are determined by the fundamental natural frequency of the plate.
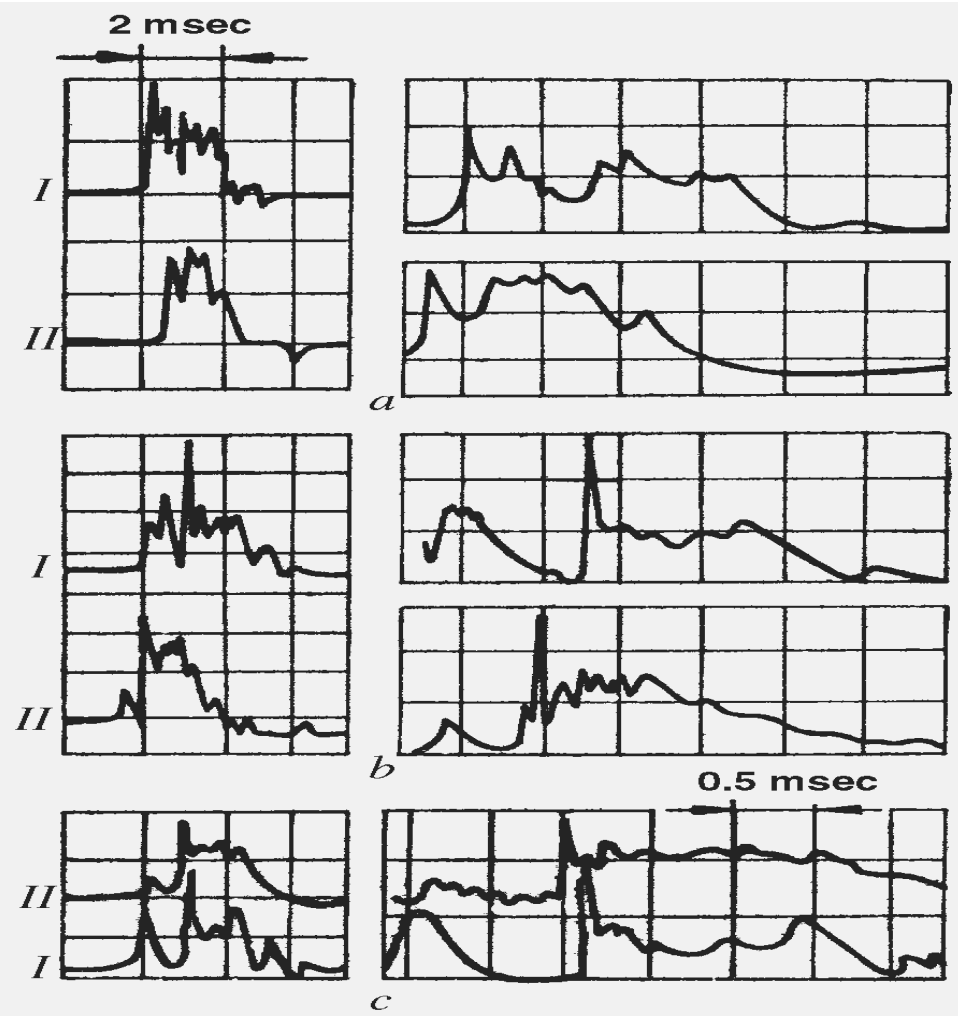

Figure 7: $\quad$ Effect of plate thickness on the water pressure on the tube wall.

There the effects of the plate thickness are weaker in oscillograms I measured further from the plate than oscillograms II. The first peak is determined by the frontal part of the incident wave. Therefore this peak depends weakly on the plate thickness. The second peak is determined by the water-plate interaction. For $h=4 \mathrm{~mm}$ (b) and $h=2 \mathrm{~mm}$ (c) the cavitation is determined by the form and the amplitude of this peak. The third peak is formed from the reverse motion of the plate and connects with the period of the natural oscillation of the plate. During the initial period of the interaction the water motion may be considered as one-dimensional. Then, because of the plate deflection, which depends strongly on the radial coordinate, the water motion near the plate transforms into two-dimensional motion, and radial waves of pressure and cavitation are formed. 


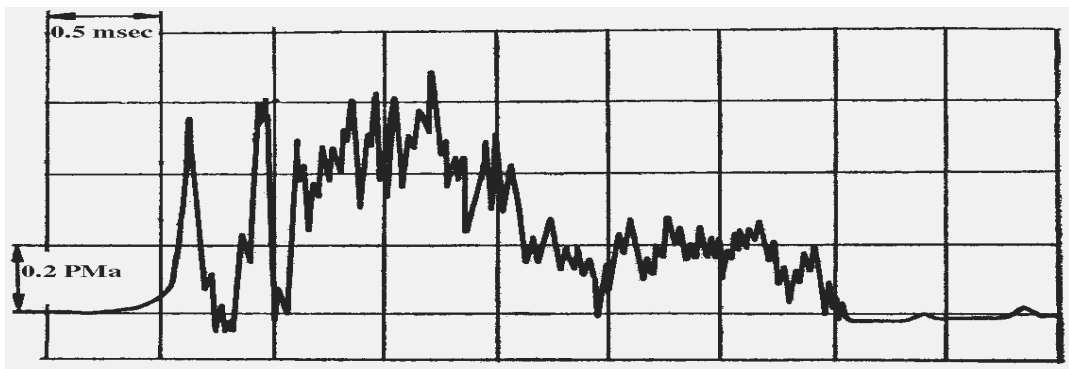

Figure 8: $\quad$ The pressure in the plate centre (aluminium alloy, $h=2 \mathrm{~mm}$ ).

Therefore oscillograms measured at different points of the plate could be expected to be different.

The oscillogram of pressure measured near the centre of the plate with $h=2$ $\mathrm{mm}$ is presented in Fig. 8. The maximum values of the pressure and the plate deflection were approximately $0.7 \mathrm{MPa}$ and $0.52 \mathrm{~mm}$ respectively. One can see that this oscillogram is quite different from ones presented in Fig. 7(c) taken at the tube surface, although the oscillograms were obtained from similar plates.

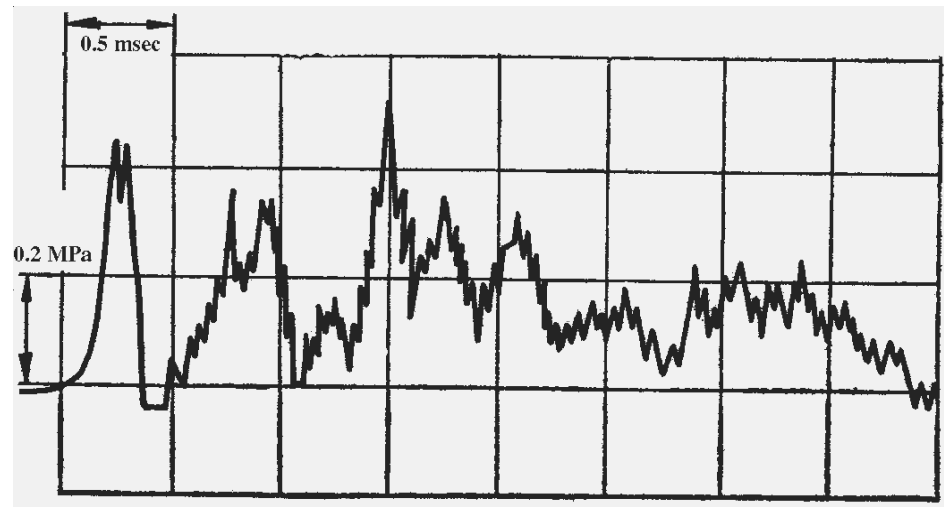

Figure 9: $\quad$ The pressure in the plate centre (bronze alloy, $h=1 \mathrm{~mm}$ ).

Various plate thicknesses and materials were chosen for the experiments so as to assure that there was sufficient deflection to cause the appearance of cavitation effects in the zone of elastic behaviour of the plate material. The pressure oscillogram for a plate from bronze alloy with a thickness $(h=1 \mathrm{~mm})$ is presented in Fig 9. The oscillogram differs from those presented in Figs. 7 and 8 as it has many peaks. These peaks are connected with strong radial motion of water along the plate during the interaction. The lifetime of the cavitation zones is determined by the intervals between the peaks on the pressure curves. The maximum lifetime at the centre of the plate is thus about 0.2 milli-s.

These figures show that the cavitation measured near the plates strongly depends on mechanical and geometrical properties of the plates. In particular, if 
the plate is thick enough, cavitation effects are either absent or small. However, if the ratio of the radius $R$ of the plate to its thickness $h$ is large, then the effect of cavitation on the resulting pressure may be very important. It is evident that the initial wave is damped by a thin plate. Therefore for thin plates the first peak of the pressure may be much smaller than the amplitude of the initial wave $(1.25$ $\mathrm{MPa}$ ). The subsequent two-dimensional waves generated by cavitation then move along the plate surface. These waves form the peaks measured in the plate centre. Hence the lifetime of the cavitation cavities depend strongly on the plate properties.

This two-dimensional cavitation, which has been shown to be strongly dependent on the plate properties, can be expected to cause hull cavitation. At the same time cavitation may be generated far from the plate due to the resulting superposition of the incident (Fig. 6) and reflected waves (Fig. 7, oscillograms I). This phenomenon is called bulk cavitation, and in these experiments, this cavitation was essentially one-dimensional.

\section{Conclusions}

The experimental results presented in this paper allow us to make the following conclusions: 1) There is similarity in the shock pressures generated by extreme surface water waves (Fig. 3) and the underwater waves (Fig. 6) on rigid thick plates; 2) In the majority of cases the elastic deformation of thin plates by a short water wave pressure pulses is accompanied by hull cavitation; 3) The cavitation zones can enclose practically the whole plate surface and completely change the water loading onto the plate, compared to the pressures that would be developed in the absence of cavitation; 4) The interaction is not a one-dimensional process. The plate deformation generates radial pressure and cavitation waves, which create and collapse the cavitation cavities; 5) Cavitation interaction of extreme water waves with structures and hull response is a complex topic, which is not well understood and is expected be important for the design of advanced ships in the future.

\section{References}

[1] Kharif, C., Pelinovsky, E. Physical mechanisms of the rogue wave phenomena. Europenian J. of Mechanics B 2003, 22, 603-634.

[2] Wang, D.W.; Mitchell, D.A.; Teague, W.J.; Jarosz, E.; Hulbert, M.S. Extreme waves under Hurricane Ivan. Science 2005, 309 (5736), 896.

[3] Smith, C.B. Extreme Waves; Joseph Henry Press: Washington, 2006.

[4] Dysthe, K., Krogstad, H.E., Müller, P. Ocean rogue waves. Annu. Rev. Fluid Mech. 40, 283-310, 2008.

[5] Clauss, G.F., Schmittner, C.E., Hennig, J., Simulation of rogue waves and their impact on marine structures In MAXWAVE Final Meeting, 8-10, October, Geneva, Switzerland, 2003.

[6] $15^{\text {th }}$ International Ship and Offshore Structures Congress 2003, August 1115, 2003, San Diego, USA. Volume 1, Dynamic Response, pp.193-264. 
[7] $16^{\text {th }}$ International Ship and Offshore Structures Congress 2006, August 20 25, 2006, Southampton, UK. Volume 2, Naval Ship Design, pp.213-263.

[8] Galiev, Sh. U. Influence of cavitation upon anomalous behavior of a plate/liquid/underwater explosion system. Int. J. Impact Engng. 1997, 19 (4), 345-359.

[9] Felippa, C.A., Park, K.C., Farhat, C. Partitional analysis of coupled mechanical systems. Report CU-CAS-99-06, Center for Aerospace Structures, College of Engineering, University of Colorado, USA, 1999.

[10] Russell, R.C.H., Macmillan, D.H. Waves and Tides. Greenwood Press, Westport, Connecticut, 1970.

[11] Peregrine, D.H. Water-wave impact on walls. Annu. Rev. Fluid Mech. 35, 23-43, 2003.

[12] Grimshaw, R., Pelinovsky, E., Talipova, T. Large-amplitude long wave interaction with a vertical wall. Europenian J. of Mechanics B 2008, 27, 409-418.

[13] Lavrenov, I.V. Wind-Wave in Ocean, Springer, 2002. 